\title{
Axial length impact on high-speed centrifugal compressor flow
}

\author{
P. Le Sausse ${ }^{1,2}$, P. Fabrie ${ }^{1} \&$ D. Arnou ${ }^{2}$ \\ ${ }^{1}$ Université de Bordeaux, IPB, UMR5251, ENSEIRB-MATMECA, \\ Talence, France \\ ${ }^{2}$ Johnson Controls Industries, France
}

\begin{abstract}
Flow computations have been performed in three different radial centrifugal compressors, focused on steady and compressible flows with steam as the fluid. Navier-Stokes equations, coupled with k- $\varepsilon$ turbulence model, are solved by the commercial software ANSYS-CFX by means of the volume finite method. The three impellers have similar blade angles, diameter and working conditions. They differ from each other by their axial length for mechanical and industrial reasons. Analysis reveals a larger recirculation cellule for flattened impellers. Two means are used to emphasize flow separation causes and consequences. First, velocity and pressure distributions are displayed along blade pressure and suction sides, which enables us to discover a large velocity difference between both sides, especially close to the impeller shroud. The velocity gap creates viscous stresses and involves an increase of turbulence kinetic energy which is clearly visible on meridian and blade-to-blade planes. Second, velocity vector projections on a circumferential plane show the presence of an undesirable secondary flow, source of vortex and flow separation. This analysis describes the impact of axial length on flow separations and efficiency.
\end{abstract}

Keywords: centrifugal compressor, radial impeller, steam, jet-wake, flow separation, recirculation, secondary flow.

\section{Introduction}

Turbocompressors are widely used in industrial applications [1]. One type of these machines is the centrifugal compressor, which consists of converting kinetic energy in to pressure energy. 


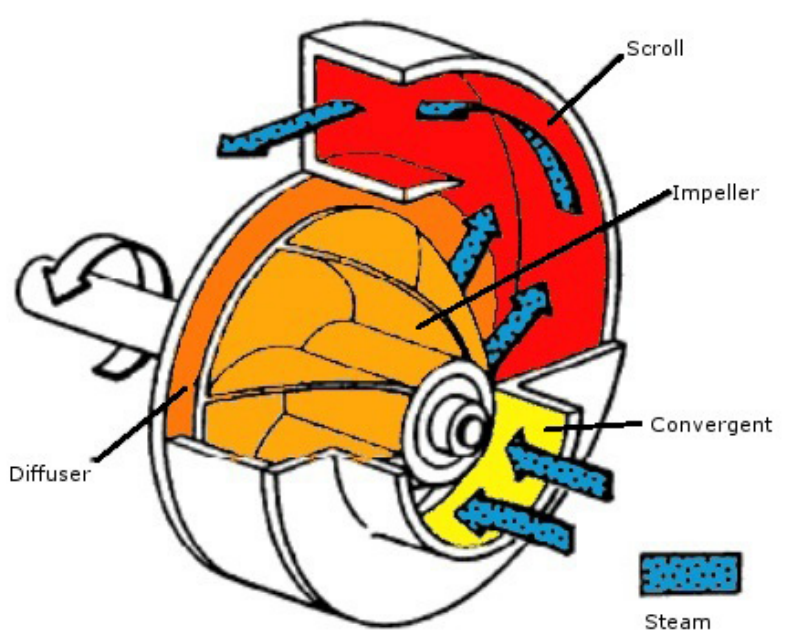

Figure 1: Compressor components.

High-speed compressors are subjected to dynamic and mechanical difficulties due to severe centrifugal restrictions. The impeller flattening enables to lighten it to reduce dynamic limitation, and also decreases mechanical strains thanks to a more suitable shape.

\section{Impeller geometry}

A centrifugal compressor, also called radial compressor, is composed of many components as indicated by figure 1 :

- the convergent axially guides fluids into the impeller;

- the impeller is a rotating part and is the main compressor element because it provides work to the fluid. The blades press the fluid which undergoes a centrifugal acceleration;

- the diffuser increases pressure energy in slowing fluid down;

- the scroll plays the same role as the diffuser and transfers fluid to other process components.

\subsection{Generalities}

We can define an impeller geometry by various parameters:

- hub and shroud profiles;

- inlet and outlet diameters;

- blade angles. 


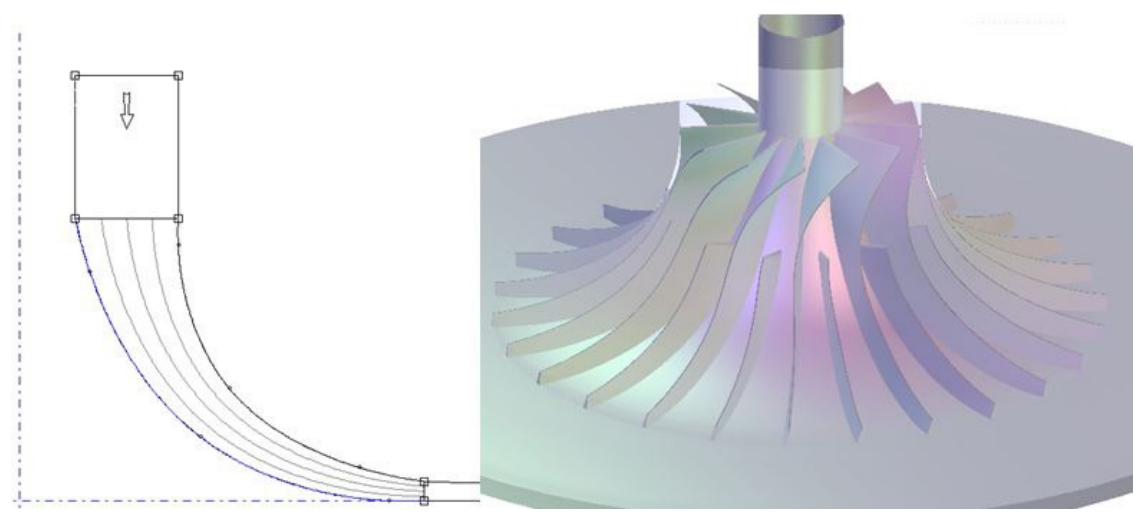

Figure 2: Impeller CCD5, with 35 percent axial length. Axial section on the left, $3 \mathrm{D}$ view on the right.

Flow is more or less impacted by each of these parameters. Choice among them is done in function of needs and specifications.

Once inlet and outlet diameters are chosen (generally for mechanical and specification reasons), blade angle is likely the most influential parameter [2] in efficiency and head. Usually, blade angle is defined by the two angles $\beta$ and $\theta$ which are distributed along blade length.

Last degrees of freedom are hub and shroud profiles that can be set without changing impeller diameter or blade angles.

\subsection{Axial length}

Axial length characterizes impeller height and is given as a percentage of impeller outlet diameter.

Reasons to modify axial length may be multiple. First, for dynamics reasons, a flattened impeller is lighter and can have a higher rotation speed. We can also guess that the shorter is the flow distance, the less are friction losses, and thereby efficiency may be improved. Inversely, in raising impeller height, we limit flow separation risks. Indeed, flow separation may appear on the shroud when it is too flattened. Moreover, by a more indirect way, a flattened impeller involves shorter blades and this can modify blade angle distributions along blade length and might affect the flow.

Figures 2 and 3 show two of the three impellers we compare in this paper: smaller and bigger axial length.

\subsubsection{Independence of axial length}

Axial length modification has an inevitable impact on blade angles, indeed blade angles are distributed along blade length. Two means are employed for blade angle distributions: 


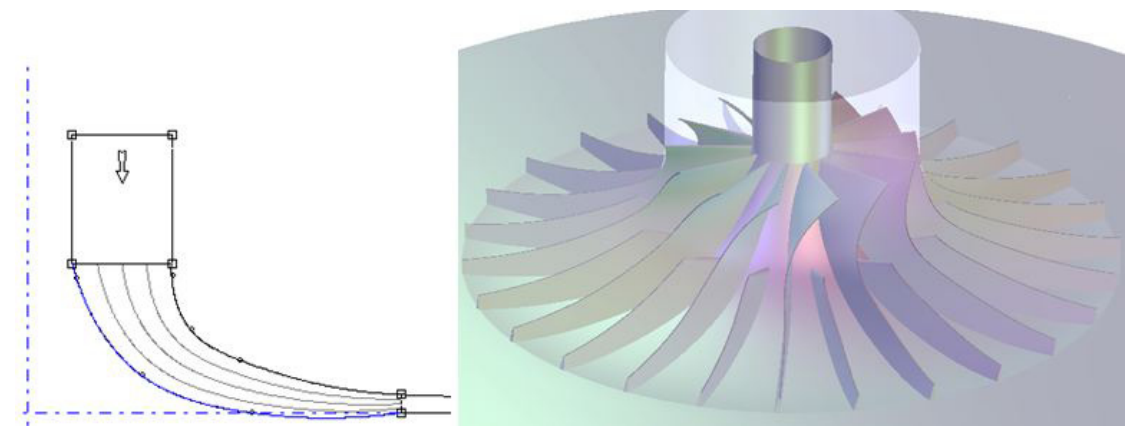

Figure 3: Impeller CCD5, with 20 percent axial length. Axial section on the left, $3 \mathrm{D}$ view on the right.

- in relation to blade length $\mathrm{M}$;

- in relation to blade length percentage $\mathrm{M} \%$.

When axial length is modified, blade length is necessarily changed also. That is why it is important to distinguish distributions in relation to the blade length between distributions in relation to the blade length percentage.

\section{Numerical simulations}

Three impellers are analyzed using the commercial computational fluid [3] dynamic (CFD) software ANSYS-CFX using a finite volume method [4].

Due to periodicity features of the impeller geometry, only one passage containing one main blade and one splitter (secondary blade) was modeled. To ensure a good transition between impeller and diffuser and to improve calculation costs, we apply the same periodicity on diffuser geometry.

The model uses a structured mesh made of hexahedrons. The mesh contains several thin layers along wall for accurate boundary layers calculation.

The boundary conditions are following:

- total pressure at the inlet;

- total temperature at the inlet;

- static pressure at the outlet;

- $5 \%$ turbulence intensity at the inlet;

- adiabatic wall with $8.5 \mu \mathrm{m}$ of roughness on impeller and diffuser;

- adiabatic wall with $0.5 \mathrm{~mm}$ of roughness on scroll.

Computations have solved steady and compressible Navier-Stokes equations, coupled with $k-\varepsilon$ turbulence model, with steam as fluid.

No experimental investigation has been done on these impellers but the model parameters (boundary conditions, physical model, mesh refinement) have been validated on an existing impeller with maximum of $2 \%$ of difference with experimental measures in compression performance (pressure ratio). That is why 


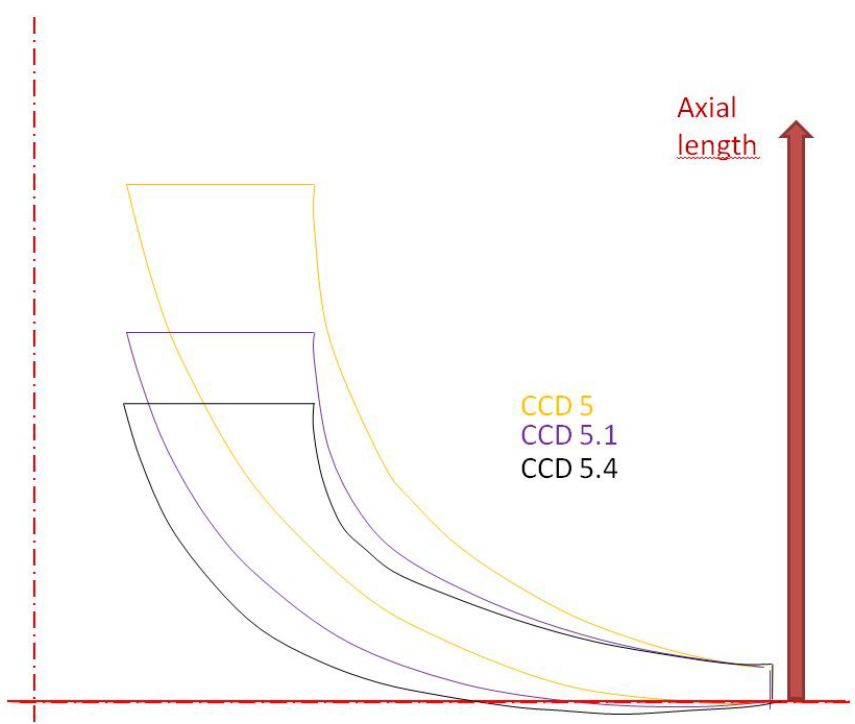

Figure 4: Profiles superimposition of the impellers.

the purpose of this paper is not to predict exact performances but only compare the flow variation when axial length is modified.

As previously described, three impellers have been compared: impellers diameter $=217 \mathrm{~mm}$;

CCD5 impeller axial length $=35 \%=76.0 \mathrm{~mm}$;

CCD5.1 impeller axial length $=25 \%=54.2 \mathrm{~mm}$;

CCD5.4 impeller axial length $=20 \%=43.4 \mathrm{~mm}$.

A superimposition of impellers profiles is shown in figure 4.

Blade lengths have been modified in flattening the impeller so blade angle distributions have been changed along blade length but it has been conserved if we consider blade length percentage as it is displayed in figure 5.

\section{Results and discussion}

\subsection{Velocity fields and distributions}

Recirculation cells characterize areas where the fluid is moving in the opposite direction of the flow. That is why it is easy to emphasize this phenomenon in displaying radial velocity as in figure 6 .

In this figure, we use ten regularly set planes, called streamwise planes, which are perpendicular to the flow.

On CCD5 impeller planes, we observe that flow is everywhere higher than in upstream plane even if we note some velocity heterogeneities. On CCD5.1 impeller, some blue areas appear, which match radial velocities close to $0 \mathrm{~m} / \mathrm{s}$. 


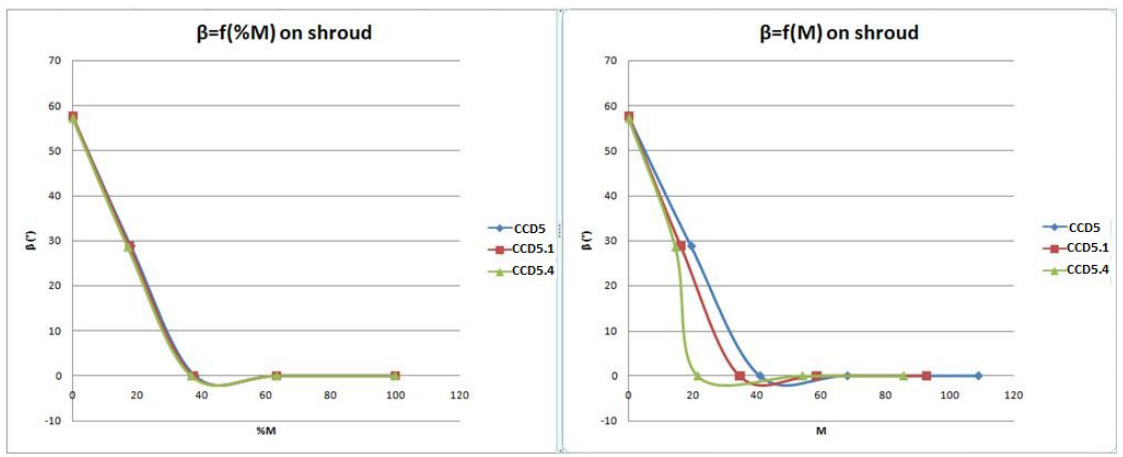

Figure 5: Blade angle distributions along blade length percentage on the left, along blade length on the right.

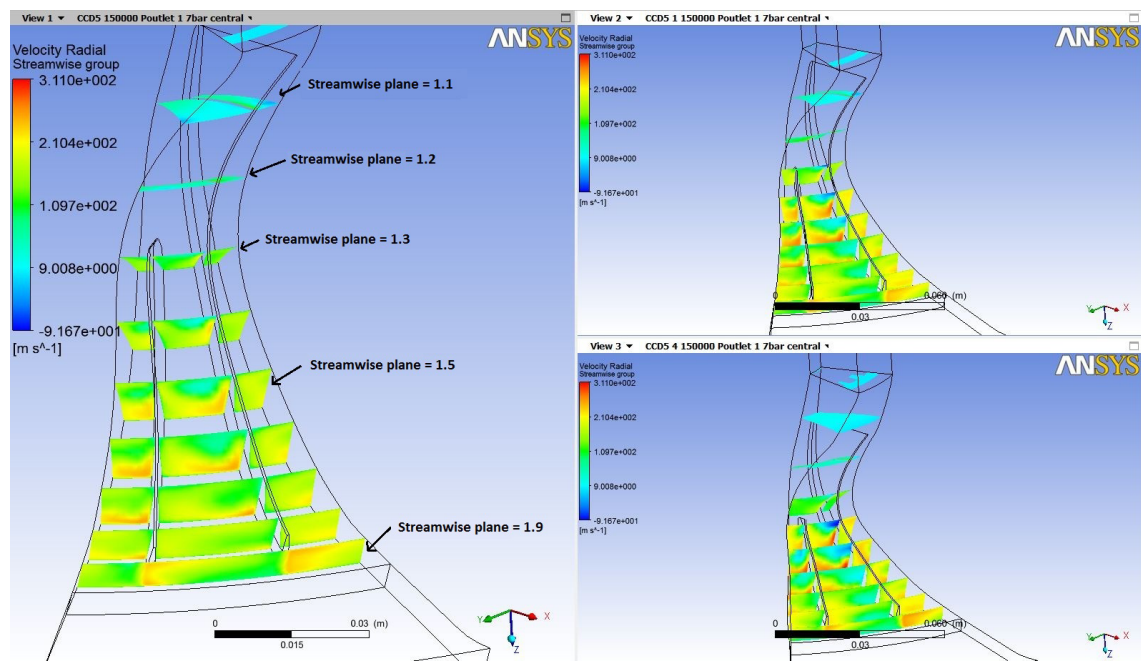

Figure 6: Radial velocity on streamwise planes CCD5 impeller on the left, CCD5.1 impeller on the top-right, CCD5.4 impeller on the bottom-right.

This velocity decrease is clearly a consequence of impeller flattening because it uprises from shroud along blade suction side. The phenomenon is accentuated on CCD5.4 impeller because we note a negative radial velocity on the same zone.

A compressor blade guides the flow and compresses the fluid in rotating. So a blade is made up of two sides: pressure side and suction side. Recirculation may appear on both sides, function of blade angle and mass flow. In our case, flow separation is bigger on suction side, that is why radial velocity distribution is displayed in figure 7 along the blade side (values are null along wall, so they are taken at the first node of the mesh). 


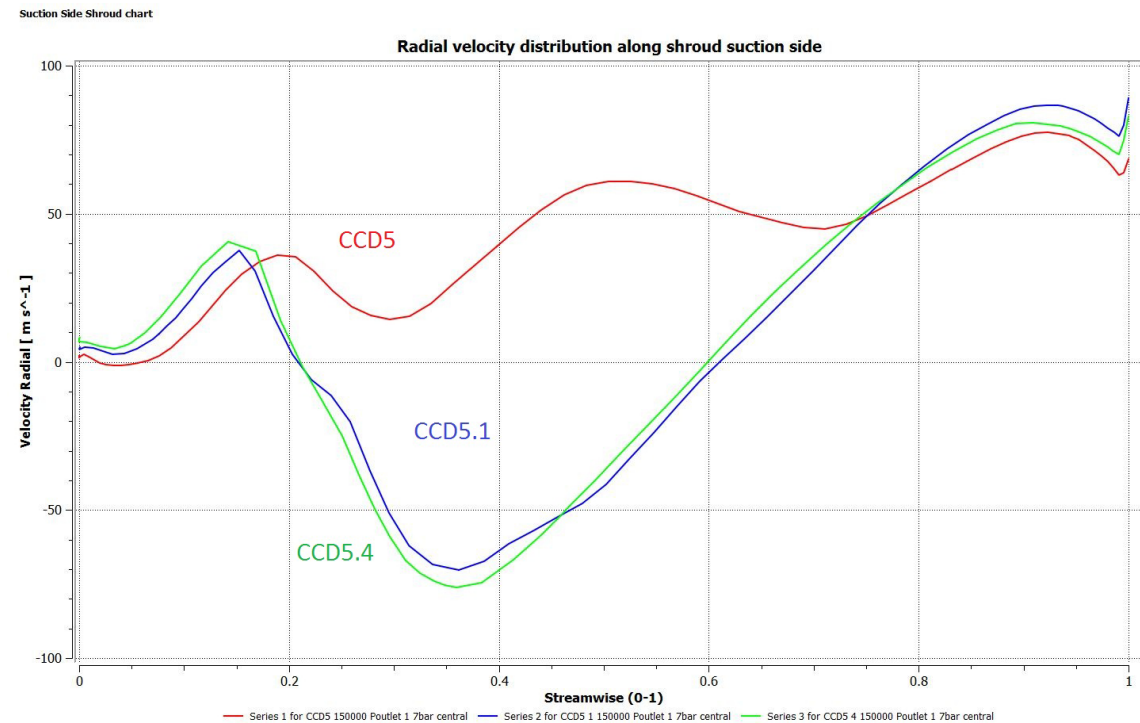

Figure 7: Radial velocity distribution along shroud suction side.

Table 1: Efficiency table.

\begin{tabular}{|c|c|c|c|}
\hline model & CCD5 & CCD5.1 & CCD5.4 \\
\hline numerical efficiency & 0.785 & 0.781 & 0.768 \\
\hline
\end{tabular}

For each impeller, we notice similar inlet and outlet velocities, which confirm the use of duplicate conditions. As on streamwise planes, a significant decrease of radial velocity for CCD5.1 and CD5.4 impellers is observed. All under zero values involve the presence of a recirculation area.

Velocity gap creates viscous stresses and involves an increase of turbulent kinetic energy which is clearly visible in figure 8 .

Turbulence kinetic energy is very low at impeller inlets and increases from flow separation first appearance. Turbulence kinetic energy maximum is twice higher in CCD5.4 model than in CCD5 one. In consequence, entropy is also upper in cases with recirculation and involves bad efficiency results as it is indicated on table 1.

\subsection{Secondary flow}

Experimental measures in impellers enabled to confirm the presence of low-momentum quantity which is coexisting with high-energy areas, called respectively wake and jet. A schema is shown in figure 9. 


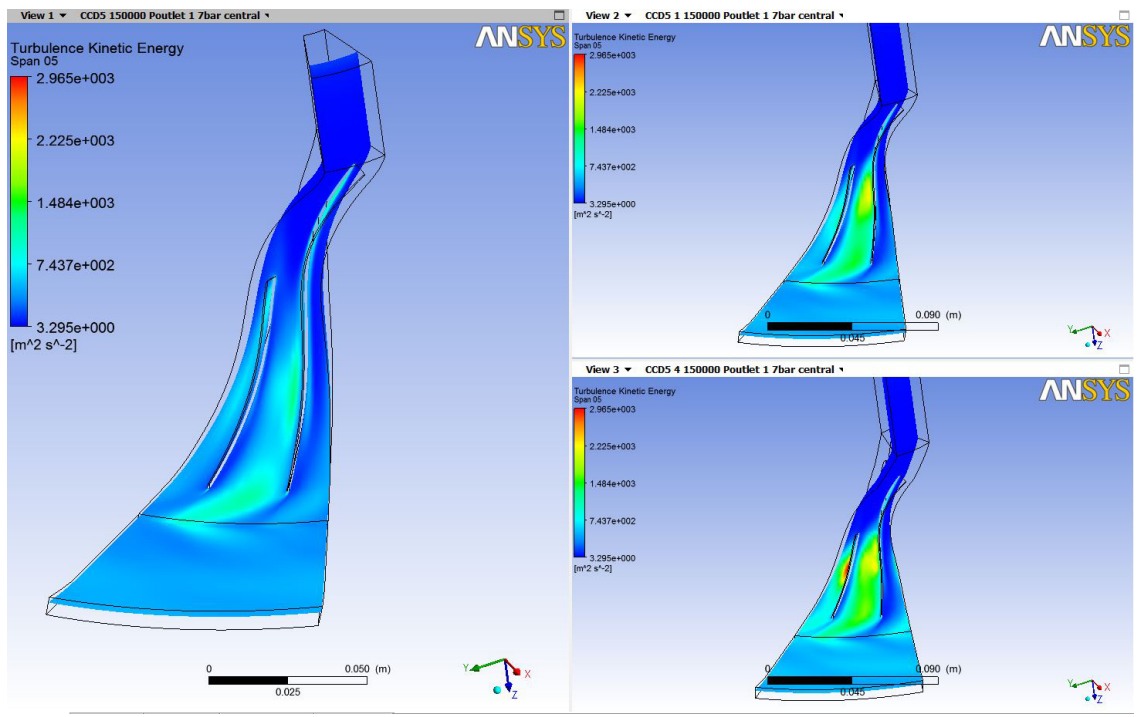

Figure 8: Turbulence kinetic energy on span=0.7 CCD5 impeller on the left, CCD5.1 impeller on the top-right, CCD5.4 impeller on the bottom-right.

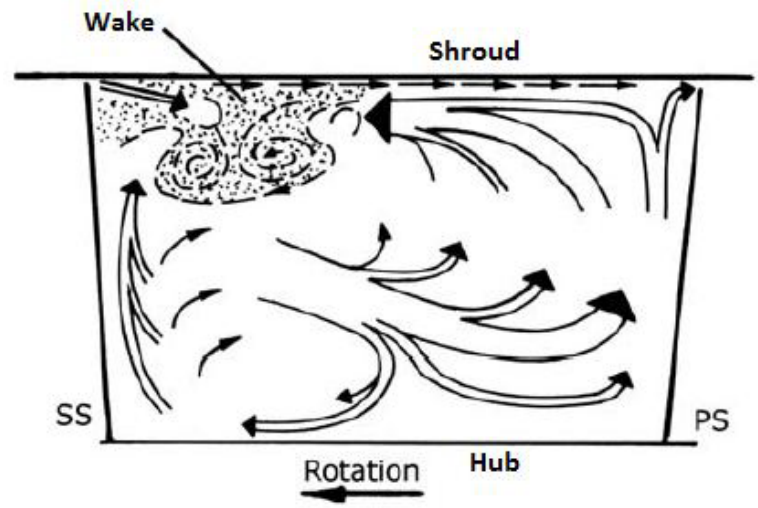

Figure 9: Schematic picture of jet-wake.

This phenomenon may be explained by flow separation on boundary layers according to Eckardt [5]. However, similar structures have been observed without significant flow separation [6] and are explained by the presence of a secondary flow due to low-momentum [7] transport close to boundary layers.

Figure 10 compares the appearance of this structure in some streamwise planes between CCD5 impeller CCD5.1 impeller. Secondary flow is indicated by black arrows. 


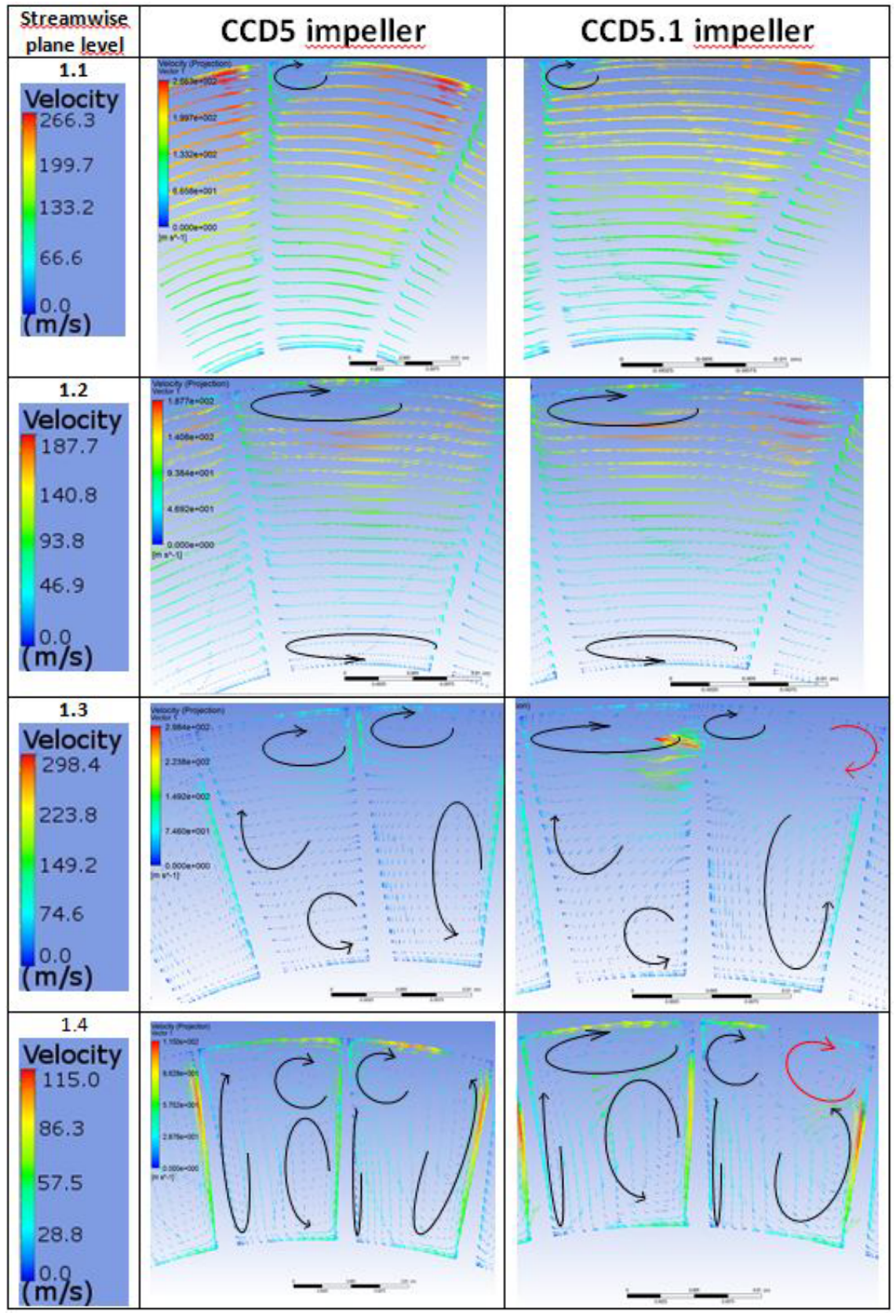

Figure 10: Velocity vector projection on streamwise planes CCD5 impeller on the left, CCD5.1 impeller on the right. 
On streamwise plane $=1.2$, we note higher velocities on CCD5.1 model than CCD5 on the top-right. Then, on streamwise plane $=1.3$, a recirculation appears on the same area on CCD5.1 whereas it does not exist on CCD5. On streamwise plane $=1.4$, this recirculation increases and it matches the same area as flow separation previously observed. These vortexes are displayed in red in the figure.

Figures 7 and 10 allow us to discover an interesting fluid acceleration before the flow separation.

\section{Conclusion}

Axial length impact on high-speed centrifugal compressor is distinctly emphasized with different means and involves the necessity to be applied with parsimoniousness.

First, we analyzed the radial velocity variable either in displaying it on suitable planes or in using distribution along blade suction side. It allows to emphasize negative values and recirculations.

Turbulence kinetic energy and entropy show the impact on losses which result in efficiency decrease.

The development of jet-wake structure enables a detailed view of the flow between blades. We can observe appearances of vortex in different impeller sections.

A flattened impeller may be required by a mechanical design but can be not optimized for aeraulic performances. Then it involves a compromise between mechanical and aeraulic design.

Moreover, this study does not enable us to know if the flow separation is due to shroud profile or blade angle distribution. In this last case, blade angle modification may improve flow quality.

\section{References}

[1] A. Javed, R.P., M. Olivero \& Buijtenen, J.V., Performance analysis of a microturbine centrifugal compressor from a manufacturing perspective. ASME Turbo Expo, 2011.

[2] Jongsik, G.A., C. Buckley, Numerical study on the effects of blade lean on high pressure centrifugal impeller performance. ASME Turbo Expo, 2011.

[3] Vierendeels, J. \& Degroote, J., Aspect of CFD Computations with commercial packages. pp. 305-328, 2009.

[4] Meauze, G., Turbomachines : calcul des écoulements incompressibles. Technique de l'ingenieur, 1982.

[5] Eckardt, D., Detailed flow investigation within a high-speed centrifugal compressor impeller. ASME Journal of fluids engineering, 1976.

[6] Ch. Hirsch, S.K. \& Pointel, G., A numerically supported investigation of the 3d flow in centrifugal impellers. part II: Secondary flow structure. ASME Turbo Expo, 1996.

[7] C. Gmelin, K.L., F. Thiele \& Meyer, R., Investigations of secondary flow suction in a high speed compressor cascade. ASME Turbo Expo, 2011. 